\title{
Does Lateral Pelvic Lymph node matters in rectal cancer
}

\author{
Ashwin Radjindrin ${ }^{1}$ and Venkatesh Shanmugam ${ }^{2}$ \\ University Hospital of North Tees, Hardwick Road, Stockton-On-Tees, TS19 8PE, UK
}

\section{Surgical Anatomy}

The lymph nodes draining the rectum below the peritoneal reflection is found to follow two major pathways (a) Along the superior rectal artery and the inferior mesenteric artery into the para-aortic nodes (b) Along the middle and inferior rectal artery into the obturator, internal iliac and external iliac nodes. The majority of the first group of nodes are resected as a part of Total Mesorectal Excision (TME) $[1,2]$. The second group of nodes, the lateral pelvic nodes, albeit being recognised long before, have triggered a significant interest in the field of rectal cancer surgery on the approach, recurrence and survival.

According to the TNM staging, involvement of the internal iliac group of nodes is considered as regional disease whereas the external and common iliac nodes are treated as metastatic disease. In contrast, the Japanese classification considers all of the lateral lymph node groups as regional nodes [3]. The Japanese guidelines for colorectal cancer (2016) recommend Lateral Lymph Node Dissection (LLND) for all tumours below the peritoneal reflection [4]. The lymphatic drainage of the rectum is depicted in the figure 1.

\section{Significance of lateral lymph node metastasis}

Synchronous lateral lymph node (LLN) disease among locally advanced rectal tumours is reported in 10-25\% [5]. According to the Japanese Society for Cancer of the Colon and Rectum data, the incidence of LLN involvement in tumours below the peritoneal reflection, with positive mesorectal nodes, is $27 \%$ [4]. Though, it appears that the reported incidence of LLN involvement varies between the East and
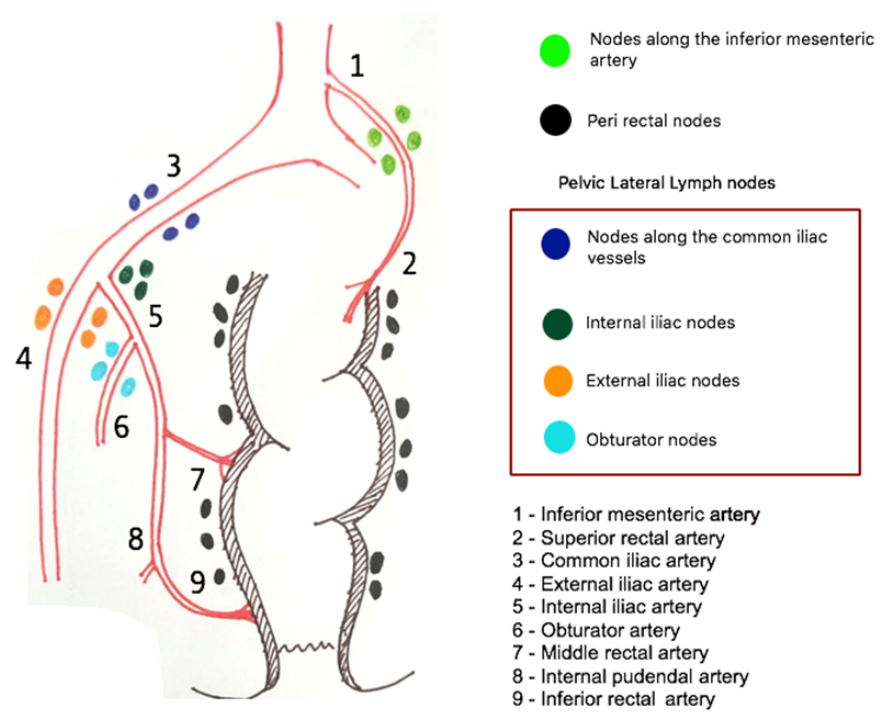

Figure 1. Lymphatic drainage of the rectum highlighting the lateral lymph nodes the West, El-Khoury et al. [6] after a detailed analysis, concluded that it might be the same (Table 1).

LLNs are also reported to be the major cause for local recurrence following curative resection. About 50\% of the local recurrences occur in the LLN basin without evidence of distant metastasis [15]. In another single-institution study conducted in Korea, $35.5 \%$ of the patients who had TME following positive response to nCRT developed local recurrence. $19.4 \%$ of them developed pelvic wall recurrence and interestingly, $16.1 \%$ developed in situ LLN metastasis, in the same site, which was suspected preoperatively [16].

\section{Managing the LLNs - differences between the east and the west}

The AJCC classifies LLN involvement as metastatic disease except for the internal iliac nodes. As the LLNs apart from the internal iliac group are considered as metastatic disease, they were dealt with less aggressively. However, radiation oncologists in the US nowadays are preferring to deal with the non-regional nodes with treatment intensification, either Radiotherapy (RT) boost or Surgical LLND [3].

The Japanese classification, on the other hand, considers all LLN involvement as regional disease, irrespective of the site. As a result, LLN dissection is routinely performed in this part of the world. The difference in approach between the East and West is shown in table 2.

\section{Advantages of LLND dissection}

According to the Japanese Society for Cancer of Colon and Rectum (JSCCR), Lateral Lymph Node Dissection (LLND) is expected to decrease the intra-pelvic recurrence by $50 \%$ and improve the 5 -year survival by $8-9 \%$ [4]. The result of a multicentre trial from Japan, JCOG2012, failed to conclude the non-inferiority of TME alone versus TME+LLND in terms of local recurrence rates. The secondary end points from the same study including the incidence of urinary and male sexual dysfunction was not found to be higher in the LLND group [17-19].

A prospective, multicentre, randomized single-blinded, phase III trial for TME versus TME + LLND for suspicious LLN involvement is currently recruiting patients at China and is expected to end by 2022 . Notably, the size of the node in MRI is used as criteria to define LLN involvement. These patients with suspicious LLN involvement are randomized and receive nCRT before proceeding to either TME or TME+LLND [5].

${ }^{\star}$ Correspondence to: Venkatesh Shanmugam, University Hospital of North Tees, Hardwick Road, Stockton-On-Tees, TS19 8PE, UK, E-mail: venkatesh. shanmugam@nhs.net

Received: October 03, 2018; Accepted: October 19, 2018; Published: October 26, 2018 
Table 1. Incidence of LLN involvement in rectal cancer

\begin{tabular}{|c|c|c|}
\hline & Incidence & Comments \\
\hline \multicolumn{3}{|c|}{ Pre-operative LLN involvement (Based on MRI) } \\
\hline Dharmarajan S et al [7] & $57 \%$ & MRI size cut off $5 \mathrm{~mm}$ \\
\hline Ishibe et al $[8]$ & $19.9 \%$ & - \\
\hline MERCURY study group [9] & $11.7 \%$ & $\begin{array}{l}\text { Based on nodal characteristics on } \\
\text { MRI. Size was not a criteria }\end{array}$ \\
\hline Ishihara et al [10] & $8.1 \%$ & Post nCRT* \\
\hline \multicolumn{3}{|c|}{ Incidence based on post-operative histopathology } \\
\hline Akiyoshi et al [11] & $40.3 \%$ & \\
\hline Moriya Y [12] & $23 \%$ & Perirectal + LLN involvement \\
\hline Ueno et at [13] & $17.3 \%$ & \\
\hline Quadros et al [14] & $17 \%$ & \\
\hline
\end{tabular}

Table 2. Differences in the understanding and management of LLNs between the East and the West

\begin{tabular}{|l|l|l|}
\hline & Western concept & Japanese concept \\
\hline Regional nodes & Internal iliac nodes & $\begin{array}{l}\text { Internal, external and common } \\
\text { iliac and obturator nodes }\end{array}$ \\
\hline Metastatic nodes & $\begin{array}{l}\text { Common iliac, external iliac and } \\
\text { obturator nodes }\end{array}$ & Not Applicable \\
\hline Management & $\begin{array}{l}\text { nCRT with RT boost to involved } \\
\text { nodes }\end{array}$ & LLN dissection \\
\hline
\end{tabular}

\section{Approaches to decrease complications in LLND}

Though the results of JCOG2012 claim that the incidence of urinary and sexual incontinence is not increased by combining LLND, they still remain as a significant morbidity following the procedure [17-19]. Laparoscopic approach is considered safer and less morbid compared to open approach [20]. Laparoscopic LLND based on vesicohypogastric fascia and uretero-hypogastric nerve fascia is found to have improved surgical outcome and decreased complication rate (Japan) [21]. Robotic LLND is feasible with better short-term outcomes and lymph node harvest. It is reported to be advantageous in male narrow pelvis [22,23].

\section{Selective Lymph node dissection}

Many studies advocate selective lymph node dissection based on various nodal characteristics on imaging pre and post-chemo radiation. Node size is commonly used as a marker and the size criteria have varied between $6 \mathrm{~mm}, 8 \mathrm{~mm}$ and $10 \mathrm{~mm}$ amongst the authors $[8,10,11]$. Rather than the size, heterogeneous MR signal and irregular border of the lymph node capsule are more reliable in predicting malignancy of the nodes on MRI [9,24]. Also, CRT significantly alters the nodal parameters in imaging that the size criteria can fail [25]. In a study, among the group that had positive response to CRT and TME subsequently, none of the nodes in follow up were more than $5 \mathrm{~mm}$ [16].

The response to CRT is also a good prognostic indicator. The subgroup of patients that had suspicious nodes but positive response to chemotherapy had better recurrence rates than the non-responders. Also, there is no significant difference in the recurrence free survival and overall survival between the non-suspicious LLN group and LLN responding to CRT. This raises the question if LLND can be reserved for the subgroup of patients with LLN disease unresponsive to CRT [26].

\section{Role of prophylactic LLND}

Tamura et al. [27] have concluded that there is no role in prophylactic LLND in stage 4 rectal cancers when there is no clinical LLN metastasis. Again, clinical LLN metastasis was defined as node $>10 \mathrm{~mm}$ in CT. There is no adequate literature so far regarding the role of prophylactic LLND. Since the JCOG2012 is only a non-inferiority trial, a future randomized clinical trial, such as a superiority study of LLND + TME versus chemo-radiotherapy + TME or LLND + chemo-radiotherapy + TME versus chemo-radiotherapy + TME, may be needed to verify the true benefit of LLND for occult lateral nodal metastases [28].

\section{Conclusion}

Although the role of prophylactic LLND is unclear, it may have a role in clinically positive lateral nodes and those that are resistant to CRT. The major question that remains still unanswered is whether routine TME + LLND post CRT in stage II and stage III rectal cancer needs to be advocated globally based on the oriental experience. The results from ongoing RCTs might provide an answer [5].

\section{References}

1. Bell S, Sasaki J, Sinclair G, Chapuis PH, Bokey EL (2009) Understanding the anatomy of lymphatic drainage and the use of blue-dye mapping to determine the extent of lymphadenectomy in rectal cancer surgery: unresolved issues. Colorectal Dis 11: 443449. [Crossref]

2. Gk K, Vd P, Ap S (2016) Is there a Role of Lateral Pelvic Lymph Node Dissection in the Current Era of Neoadjuvant Chemoradiotherapy for Rectal Cancer? J Gastrointest Dig Syst 6: 1-4.

3. Yahya JB, Herzig DO, Farrell MJ, Degnin CR, Chen Y, et al. (2018) Does a fine line exist between regional and metastatic pelvic lymph nodes in rectal cancer-striking discordance between national guidelines and treatment recommendations by US radiation oncologists. J Gastrointest Oncol 9: 441-447. [Crossref]

4. Watanabe T, Muro K, Ajioka Y, Hashiguchi Y, Ito Y, et al. (2018) Japanese Society for Cancer of the Colon and Rectum (JSCCR) guidelines 2016 for the treatment of colorectal cancer. Int J Clin Oncol 23: 1-34. [Crossref]

5. Wei M, Wu Q, Fan C, Li Y, Chen X, et al. (2016) Lateral pelvic lymph node dissection after neoadjuvant chemo-radiation for preoperative enlarged lateral nodes in advanced low rectal cancer: study protocol for a randomized controlled trial. Trials17: 561. [Crossref]

6. El-Khoury T, Solomon MJ, Young JM (2008) The incidence of lateral pelvic side-wall nodal involvement in low rectal cancer may be similar in Japan and the West (Br J Surg 2008; 95: 33-49). Br J Surg 95: 801-802. [Crossref]

7. Dharmarajan S, Shuai D, Fajardo AD, Birnbaum EH, Hunt SR, et al. (2011) Clinically enlarged lateral pelvic lymph nodes do not influence prognosis after neoadjuvant therapy and TME in stage III rectal cancer. J Gastrointest Surg 15: 1368-1374. [Crossref]

8. Ishibe A, Ota M, Watanabe J, Suwa Y, Suzuki S, et al. (2016) Prediction of Lateral Pelvic Lymph-Node Metastasis in Low Rectal Cancer by Magnetic Resonance Imaging. World J Surg 40: 995-1001. [Crossref]

9. MERCURY Study Group, Shihab OC, Taylor F, Bees N, Blake H, Jeyadevan N, et al. (2011) Relevance of magnetic resonance imaging-detected pelvic sidewall lymph node involvement in rectal cancer. Br J Surg 98: 1798-804. [Crossref]

10. Ishihara S, Kawai K, Tanaka T, Kiyomatsu T, Hata K, et al. (2017) Oncological Outcomes of Lateral Pelvic Lymph Node Metastasis in Rectal Cancer Treated with Preoperative Chemoradiotherapy. Dis Colon Rectum 60: 469-76. [Crossref]

11. Akiyoshi T, Matsueda K, Hiratsuka M, Unno T, Nagata J, et al. (2015) Indications for Lateral Pelvic Lymph Node Dissection Based on Magnetic Resonance Imaging Before and After Preoperative Chemoradiotherapy in Patients with Advanced Low-Rectal Cancer. Ann Surg Oncol 22: S614-20. [Crossref]

12. Moriya Y (2013) Treatment of lateral pelvic nodes metastases from rectal cancer: the future prospective. G Chir 34: 245-248. [Crossref]

13. Ueno M, Oya M, Azekura K, Yamaguchi T, Muto T (2005) Incidence and prognostic significance of lateral lymph node metastasis in patients with advanced low rectal cancer. Br J Surg 92: 756-763. [Crossref]

14. Quadros CA, Falcão MF, Carvalho ME, Ladeia PA, Lopes A (2012) Metastases to retroperitoneal or lateral pelvic lymph nodes indicated unfavorable survival and high pelvic recurrence rates in a cohort of 102 patients with low rectal adenocarcinoma. $J$ Surg Oncol 106: 653-658. [Crossref]

15. Yun J-A, Huh JW, Kim HC, Park YA, Cho YB, et al. (2016) Local recurrence after curative resection for rectal carcinoma: The role of surgical resection. Medicine (Baltimore) 95: e3942. [Crossref] 
16. Kim HJ, Choi G-S, Park JS, Park SY, Cho SH, et al. (2017) Optimal treatment strategies for clinically suspicious lateral pelvic lymph node metastasis in rectal cancer. Oncotarget 8: 100724-100733. [Crossref]

17. Ito M, Kobayashi A, Fujita S, Mizusawa J, Kanemitsu Y, et al. (2018) Urinary dysfunction after rectal cancer surgery: Results from a randomized trial comparing mesorectal excision with and without lateral lymph node dissection for clinical stage II or III lower rectal cancer (Japan Clinical Oncology Group Study, JCOG0212). Eur J Surg Oncol 44: 463-468. [Crossref]

18. Fujita S, Akasu T, Mizusawa J, Saito N, Kinugasa Y, et al. (2012) Postoperative morbidity and mortality after mesorectal excision with and without lateral lymph node dissection for clinical stage II or stage III lower rectal cancer (JCOG0212): results from a multicentre, randomised controlled, non-inferiority trial. Lancet Oncol 13: 616-621. [Crossref]

19. Saito S, Fujita S, Mizusawa J, Kanemitsu Y, Saito N, et al. (2016) Male sexual dysfunction after rectal cancer surgery: Results of a randomized trial comparing mesorectal excision with and without lateral lymph node dissection for patients with lower rectal cancer: Japan Clinical Oncology Group Study JCOG0212. Eur J Surg Oncol 42: 1851-1858. [Crossref]

20. Nagayoshi K, Ueki T, Manabe T, Moriyama T, Yanai K, et al. (2016) Laparoscopic lateral pelvic lymph node dissection is achievable and offers advantages as a minimally invasive surgery over the open approach. Surg Endosc 30: 1938-1947. [Crossref]

21. Matsumoto A, Arita K (2017) A technique of laparoscopic lateral pelvic lymph node dissection based on vesicohypogastric fascia and ureterohypogastric nerve fascia for advanced low rectal cancer. Surg Endosc 31: 945-948. [Crossref]
22. Kagawa H, Kinugasa Y, Shiomi A, Yamaguchi T, Tsukamoto S, et al. (2015) Roboticassisted lateral lymph node dissection for lower rectal cancer: short-term outcomes in 50 consecutive patients. Surg Endosc 29: 995-1000. [Crossref]

23. Kim HJ, Choi G-S, Park JS, Park SY, Lee HJ, et al. (2018) Selective lateral pelvic lymph node dissection: a comparative study of the robotic versus laparoscopic approach. Surg Endosc 32: 2466-2473. [Crossref]

24. Balyasnikova S, Brown G (2016) Optimal Imaging Strategies for Rectal Cancer Staging and Ongoing Management. Curr Treat Options Oncol 17: 32. [Crossref]

25. Koh DM, Chau I, Tait D, Wotherspoon A, Cunningham D, et al. (2008) Evaluating Mesorectal Lymph Nodes in Rectal Cancer Before and After Neoadjuvant Chemoradiation Using Thin-Section T2-Weighted Magnetic Resonance Imaging. Int $J$ Radiat Oncol Biol Phys 71: 456-461. [Crossref]

26. Kim MJ, Chan Park S, Kim TH, Kim DY, Kim SY, et al. (2016) Is lateral pelvic node dissection necessary after preoperative chemoradiotherapy for rectal cancer patients with initially suspected lateral pelvic node? Surgery 160: 366-76. [Crossref]

27. Tamura H, Shimada Y, Kameyama H, Yagi R, Tajima Y, et al. (2017) Prophylactic lateral pelvic lymph node dissection in stage IV low rectal cancer. World $J$ Clin Oncol 8: 412-419. [Crossref]

28. Kanemitsu Y, Komori K, Shida D, Ochiai H, Tsukamoto S, et al. (2017) Potential impact of lateral lymph node dissection (LLND) for low rectal cancer on prognoses and local control: A comparison of 2 high-volume centers in Japan that employ different policies concerning LLND. Surgery 162: 303-314. [Crossref]

Copyright: (C2018 Radjindrin A. This is an open-access article distributed under the terms of the Creative Commons Attribution License, which permits unrestricted use, distribution, and reproduction in any medium, provided the original author and source are credited. 